\title{
Applicability of an in-House Saponin-Based Extraction Method in Bruker Biotyper Matrix-Assisted Laser Desorption/Ionization Time-of-Flight Mass Spectrometry System for Identification of Bacterial and Fungal Species in Positively Flagged Blood Cultures
}

OPEN ACCESS

Edited by:

Ying Zhang,

Johns Hopkins University, USA

Reviewed by:

Kit Hang Gilman Siu,

Hong Kong Polytechnic University,

China

Yunlong $L i$

Wadsworth Center, USA

${ }^{*}$ Correspondence:

Po-Ren Hsueh

hsporen@ntu.edu.tw

Specialty section:

This article was submitted to

Infectious Diseases,

a section of the journal

Frontiers in Microbiology

Received: 22 March 2016

Accepted: 29 August 2016

Published: 15 September 2016

Citation:

Chien J-Y, Lee T-F, Du S-H, Teng S-H, Liao C-H, Sheng $W-H$, Teng L-J and Hsueh P-R (2016) Applicability of an in-House Saponin-Based Extraction Method in Bruker Biotyper Matrix-Assisted Laser Desorption/Ionization Time-of-Flight Mass Spectrometry System for Identification of Bacterial and Fungal Species in Positively Flagged Blood Cultures. Front. Microbiol. 7:1432.

doi: 10.3389/fmicb.2016.01432
Jung-Yien Chien 1,2, Tai-Fen Lee ${ }^{3,4}$, Shin-Hei Du ${ }^{3}$, Shih-Hua Teng ${ }^{5,6}$, Chun-Hsing Liao ${ }^{7}$, Wang-Hui Sheng ${ }^{2}$, Lee-Jene Teng ${ }^{3,4}$ and Po-Ren Hsueh ${ }^{2,3 *}$

${ }^{1}$ Graduate Institute of Clinical Medicine, College of Medicine, National Taiwan University, Taipei, Taiwan, ${ }^{2}$ Departments of Internal Medicine, National Taiwan University Hospital, College of Medicine, National Taiwan University, Taipei, Taiwan, ${ }^{3}$ Departments of Laboratory Medicine, National Taiwan University Hospital, College of Medicine, National Taiwan University, Taipei, Taiwan, ${ }^{4}$ Departments of Graduate Institute of Clinical Laboratory Sciences and Medical Biotechnology, National Taiwan University, Taipei, Taiwan, ${ }^{5}$ Department of Graduate Institute of Biomedical Sciences, Chang Gung University, Tao-Yuan, Taiwan, ${ }^{6}$ Bruker Taiwan Co., Ltd., Taipei, Taiwan, ${ }^{7}$ Department of Internal Medicine, Far Eastern Memorial Hospital, Taipei, Taiwan

We used an in-house saponin-based extraction method to evaluate the performance of the Bruker Biotyper matrix-assisted laser desorption/ionization time-of-flight mass spectrometry (MALDI-TOF/MS) system for the identification of bacteria and fungi in 405 positively flagged blood culture bottles. Results obtained from MALDI-TOF/MS were compared with those obtained using conventional phenotypic identification methods. Of the 405 positively flagged blood culture bottles, 365 showed monomicrobal growth and were correctly identified to the species (72.1\%) or genus (89.6\%) level using the Bruker Biotyper system. The remaining 40 positively flagged blood culture bottles showed polymicrobial growth. Of them, $82.5 \%(n=33)$ of the isolates were correctly identified to the species level and $92.5 \%(n=37)$ to the genus level using the Bruker Biotyper system. The overall accuracy of identification to the genus level in flagged blood cultures was 89.5\% for Gram-positive organisms, 93.5\% for Gram-negative pathogens and $71.9 \%$ for fungi. Confidence scores were $\geq 1.500$ for 307 (75.8\%) bottles, $\geq 1.700$ for 249 (61.5\%) bottles and $\geq 2.000$ for 142 (35.1\%) bottles. None of the yeast cultures yielded scores $\geq 1.700$. Using an identification-score cutoff of $\geq 1.500$, the MALDI Biotyper correctly identified $99.2 \%$ of Gram-positive bacteria, 97.6\% of Gram-negative bacteria and $100 \%$ of yeast isolates to the genus level and $77.6 \%$ of Gram-positive bacteria, $87.1 \%$ of Gram-negative bacteria and $100.0 \%$ of yeast isolates to the species level. The overall rate of identification using our protocol was 89.9\% (364/405) for genus level 
identification and $73.1 \%(296 / 405)$ for species level identification. Yeast isolates yielded the lowest confidence scores, which compromised the accuracy of identification. Further optimization of the protein extraction procedure in positive blood cultures is needed to improve the rate of identification.

Keywords: MALDI-TOF MS, Bruker Biotyper system, Vitek MS system, flagged blood cultures, performance

\section{INTRODUCTION}

Bloodstream infections are a leading cause of admission to intensive care units and carry a high mortality rate. Identification of the causative microorganism(s) is central to the treatment of bloodstream infections, and clinical outcome can be greatly improved by the timely administration of appropriate antimicrobial agents (Deen et al., 2012; Lai et al., 2014; Bassetti et al., 2015).

Matrix-assisted laser desorption/ionization time-of-flight mass spectrometry (MALDI-TOF/MS) techniques are now routinely used for the direct identification of microorganisms from agar cultures and positively flagged blood cultures (Moussaoui et al., 2010; Lagace-Wiens et al., 2012; Martiny et al., 2012). These techniques provide definitive identification of pathogens causing bloodstream infections $18-48 \mathrm{~h}$ earlier than conventional methods. Identification of pathogens using the MALDI-TOF/MS system has markedly reduced the rate of administering improper antimicrobial agents, has contributed to decreased morbidity and mortality rates and is associated with reduced hospital costs for patients with bloodstream infections (Loonen et al., 2012; Martiny et al., 2013). A number of purification methods are available to prepare samples for MALDI-TOF/MS analysis such as differential centrifugation, lysis centrifugation, pre-incubation on sold media and the SepsiTyper $^{\text {TM }}$ kit (Schubert et al., 2011; Saffert et al., 2012; March-Rossello et al., 2013). Of those methods, processing of specimens using the SepsiTyper ${ }^{\mathrm{TM}}$ kit has been shown to result in highly accurate identification rates; however, the kit is not widely used because of its high cost (Buchan et al., 2012; Lagace-Wiens et al., 2012).

In this study, we used an in-house saponin-based extraction method that was modified from a previous study (Martiny et al., 2012) to evaluate the performance of the Bruker Biotyper (Bruker Daltonics) MALDI TOF-MS system for the identification of bacteria and fungi in positively flagged blood culture bottles.

\section{MATERIALS AND METHODS}

\section{Blood Cultures}

All positive cultures preserved in Bactec Plus Aerobic/F bottles or Bactec Anaerobic Lytic/10 bottles (Becton-Dickinson Microbiology Systems, Sparks, MD, USA) that had been obtained from patients treated for bloodstream infections at the National Taiwan University Hospital (NTUH) during the period October 13, 2014 to January 15, 2015 were evaluated by conventional phenotypic methods and the MALDI TOF-MS Biotyper system. For each patient, only the first positive blood culture broth was included in the study. Time to positivity (TTP) of each positively flagged blood cultures was evaluated. Microbial identification using the MALDI Biotyper was performed directly from positive blood culture broth and from subsequent colonies on Tryticase soy agar with $5 \%$ sheep blood agar (BAP) or chocolate agar after overnight culture.

\section{Processing of Flagged Positive Blood Cultures by the MALDI Biotyper}

The in-house saponin-based extraction method used in this study followed the protocol described by Martiny et al. with slight modifications (Martiny et al., 2012). Briefly, $1 \mathrm{~mL}$ of positive blood sample was added to $200 \mu \mathrm{L}$ of a $5 \%$ saponin lysis solution. The tube was thoroughly vortexed for $10 \mathrm{~s}$ and then centrifuged for $1 \mathrm{~min}$ at $13,000 \mathrm{~g}$. The supernatant was discarded, the pellet was repeatedly washed by pipetting with $1 \mathrm{~mL}$ of de-ionized water and then the solution was centrifuged for $1 \mathrm{~min}$ at $13,000 \mathrm{~g}$. The supernatant was discarded and the pellet was subjected to a formic acid extraction method for MALDI Biotyper analysis as described below. All microbial species reported by the MALDI Biotyper were recorded even when identification score values were $<2.000$ (genus-level identification or no reliable identification).

\section{Performance of the MALDI Biotyper from Subcultured Colonies}

For analysis by the MALDI Biotyper system on BAP, two to three colonies were transferred to a 1.5-ml screw-cap Eppendorf tube containing $300 \mu \mathrm{l}$ of distilled water and then mixed with $900 \mu \mathrm{l}$ of ethanol by pipetting. The suspension was pelleted by centrifugation at 13,000 rpm for $2 \mathrm{~min}$, evaporated to dryness, and then reconstituted in $50 \mu \mathrm{l}$ of $70 \%$ formic acid. After incubation for $30 \mathrm{~s}, 50 \mu \mathrm{l}$ of acetonitrile (Sigma-Aldrich) was added. The suspension was then centrifuged at 13,000 rpm for $2 \mathrm{~min}$. Then, $1.0 \mu \mathrm{l}$ of the supernatant was applied to a 96-spot polished steel target plate (Bruker Daltonik GmbH, Bremen, Germany) and dried. A saturated solution of $1.0 \mu \mathrm{l}$ of MALDI matrix (HCCA; Bruker Daltonik GmbH, Bremen, Germany) was applied to each sample and dried. Measurements were performed with the Bruker Microflex ${ }^{\text {TM }}$ LT MALDI-TOF MS system (Bruker Daltonik GmbH, Bremen, Germany) using FlexControlTM software with Compass Flex Series version 1.3 software and a $60 \mathrm{~Hz}$ nitrogen laser (337 $\mathrm{nm}$ wavelength). Spectra were collected in the linear positive mode in a mass range covering 1960-20,132 $\mathrm{m} / \mathrm{z}$. Spectra ranging from the mass-to-charge ratio $(\mathrm{m} / \mathrm{z})$ 2000-20,000 were analyzed using Bruker Biotyper automation control and the Bruker Biotyper 3.1 software and library (DB 5627 with 5627 entries). Identification scores of $\geq 2.000$ indicated species-level identification, scores 
ranging from 1.700 to 1.999 indicated genus-level identification, and scores of $<1.700$ indicated no reliable identification. All isolates with discrepant identification results between phenotypic and Bruker Biotyper methods were retested twice.

\section{Species Identification by Conventional Phenotypic Methods}

Two commercial biochemical identification systems, namely the Vitek 2 system (bioMe'rieux, Marcy l'Etoile, France) and the Phoenix system (Becton-Dickinson Microbiology Systems), were routinely used for species identification of positive blood cultures. The Phoenix system NMIC/ID-72 and PMIC/ID30 were used for identification of Streptococcus species and Aeromonas species, respectively. For identification of other bacterial organisms, the Vitek 2 system (Vitek 2 GN and Vitek 2 GP cards) was used. For identification of yeasts, the Vitek 2 yeast identification card (Vitek 2 Yeast ID) was used.

\section{Comparison of Identification Results}

The clinical microbiology laboratory at the NTUH routinely reported the identification results based on those obtained by conventional phenotypic identification methods. In this study, the identification results obtained by the MALDI Biotyper system from positive blood culture bottles were compared with those obtained by the MALDI Biotyper and conventional phenotypic identification methods from sub-cultured colonies. We used the identification results by the MALDI Biotyper from subcultured growth colonies to resolve the discrepancy between results by the MALDI Biotyper system directly from positive blood culture bottles and conventional phenotypic identification methods. The rates of concordant results for identification of organisms to the genus and species levels were calculated. Types of identification (I-IX) were defined based on the results of identification to the species or genus level among three methods (Figure 1).

\section{Turn-around Time (TAT) of Blood Cultures}

TAT of blood cultures was defined as the time interval between collection of blood from patients and identification results from positive blood cultures reported by the central laboratory. The maximum time allowed to collect blood samples, transport the blood culture bottles to the laboratory, and upload the culture bottles into the Bactec FX system was $2 \mathrm{~h}$. Following the alarming of positive blood cultures, around $2 \mathrm{~h}$ was need to stain the positive culture broths, prepare and perform the MALDI-TOF MS analysis (around $30 \mathrm{~min}$ ), and report the identification results via laboratory and hospital information systems. The

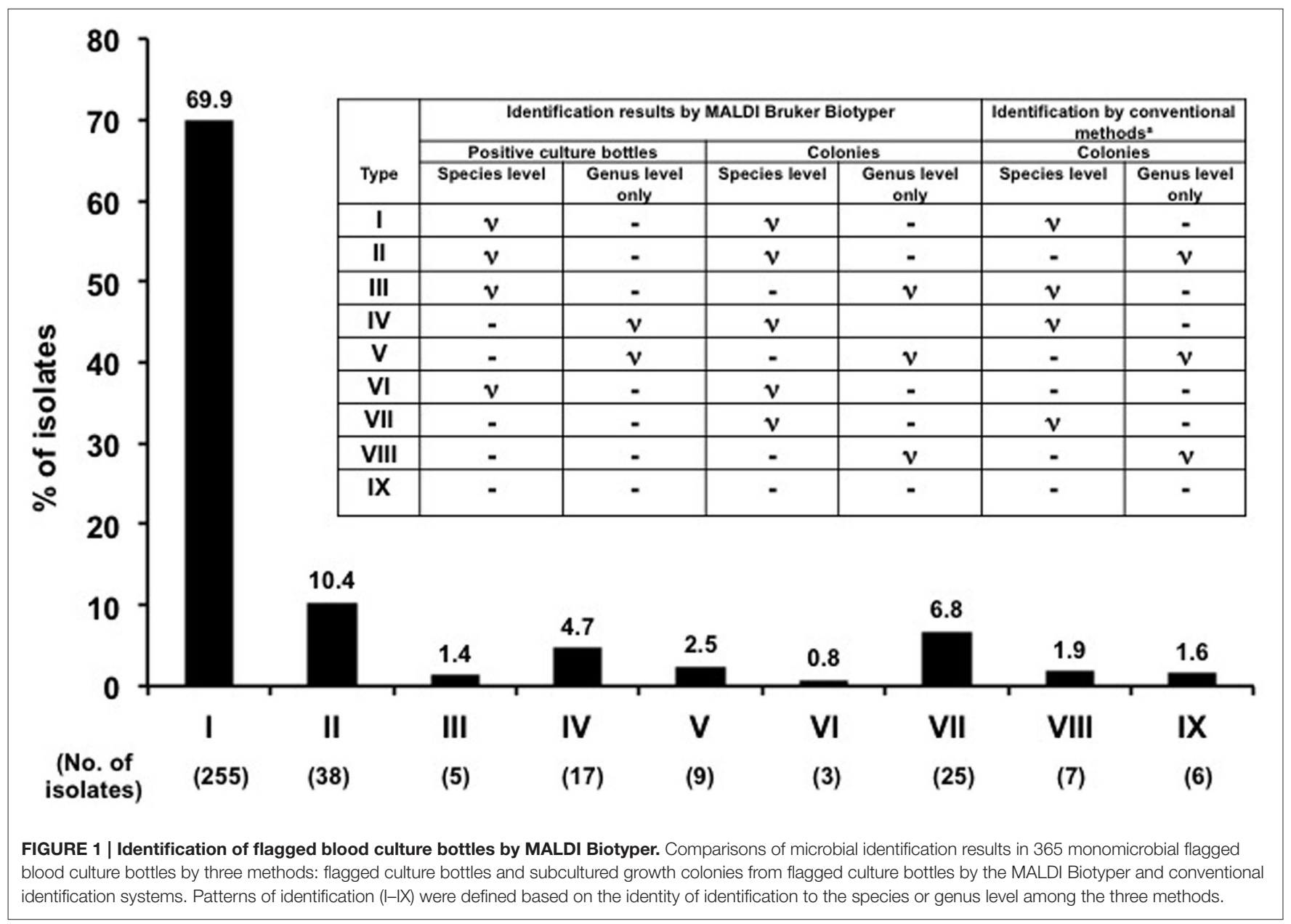


TAT to identify specific organisms directly from flagged blood cultures by the MALDI Biotyper was calculated as the average TTP for specific organisms in positive blood cultures plus $4 \mathrm{~h}$. The TAT to identify specific organisms isolated from blood cultures identified by the MALDI Biotyper and conventional phenotypic identification methods from sub-cultured colonies was $16-24 \mathrm{~h}$ longer than the TAT to identify specific organisms in flagged blood cultures using the MALDI Biotyper system.

\section{RESULTS}

During the study period, a total of 405 positive blood cultures, including 365 monomicrobal and 40 polymicrobial positive blood cultures that had been determined by the conventional identification systems were evaluated by MALDI-TOF MS.

\section{Discrepancy Analysis}

Table 1 shows the TTP data and rates of concordant identification to the genus and species levels for 365 monomicrobial blood cultures in flagged culture bottles and subcultured colonies by the MALDI Biotyper and by conventional phenotypic identification methods. All isolates of Staphylococcus aureus $(n=23)$, Enterococcus faecalis $(n=6)$, and Pseudomonas aeruginosa $(n=8)$ were correctly identified by the phenotypic methods and MALDI-TOF. Greater than $90 \%$ concordance was obtained for Escherichia coli $(n=69)$ and Klebsiella pneumoniae $(n=31)$. Among the 29 isolates of Candida species, $21(72.4 \%)$ were identified to the genus level and $13(44.8 \%)$ were identified to the species level in flagged culture bottles by the MALDI Biotyper and by conventional phenotypic identification methods in subcultured colonies. Only one of seven isolates of $C$. tropicalis in flagged culture bottles was identified correctly by the MALDI Biotyper.

One isolate from a flagged positive culture bottle was incorrectly identified as $S$. aureus by the MALDI Biotyper (identification score, 1.006); however, that isolate was correctly identified as Micrococcus species from subcultured colonies by the MALDI Biotyper (identification score, 2.181) and the Vitek 2 system. One isolate from a flagged positive culture bottle was incorrectly identified as $K$. pneumoniae by the MALDI Biotyper (identification score, 1.185); however, that isolate was subsequently correctly identified as E. coli from subcultured colonies by the MALDI Biotyper (identification score, 2.247) and the Vitek 2 system. Two isolates of S. maltophilia in positive blood culture bottles were correctly identified by the MALDI Biotyper.

\section{Monomicrobial Growth}

Table 2 summarizes 37 incorrect identification results (types of identification VII, VIII, and IX) in monomicrobial growth colonies by the MALDI Biotyper in flagged blood culture bottles in comparison with those by MALDI Biotyper in subculture growth colonies from flagged culture bottles and conventional identification systems. The identification results obtained by the MALDI Biotyper and the conventional identification system in subcultured growth colonies were $83.8 \%$ compatible at the species level $(n=23)$ and the genus level $(n=8)$.
In general, among the monomicrobal positive blood cultures, the MALDI Biotyper identified 89.6\% (identification types I-V) of organisms directly from positive blood cultures to the species $(72.1 \%)$ or genus levels compared with by conventional phenotypic methods from sub-cultured colonies of positive blood cultures (Figure 1).

\section{Polymicrobial Growth}

Among the 40 flagged blood culture bottles with polymicrobial growth, two organisms were isolated from 38 (95\%) of the bottles and three organisms were isolated from two (5\%) of the bottles (Table 3). Among these 40 flagged blood culture bottles, the MALDI Biotyper correctly identified one of the isolated organisms to the species level in 33 (82.5\%) bottles and to the genus level in 37 (92.5\%). The results of one bottle (no. 25) identified by the MALDI Biotyper as containing Aeromonas jandaei (best-matched organism identified) and E. coli (second-matched organism identified) were compatible with the identification results obtained by conventional phenotypic methods. The results of three bottles (no. 7, 22, and 28) identified by the MALDI Biotyper as comprising Acinetobacter nosocomialis, Aromatoleum terpenicum, and K. pneumonia, respectively, differed from those obtained by the phenotypic methods. The results of species identification by the MALDI Biotyper (best-match organisms) from 34 bottles (85.0\%) and from colonies on BAP or chocolate agar sub-cultured from positive blood culture bottles were identical to those obtained by conventional phenotypic methods.

\section{Concordant Identification and TAT}

Table 4 shows that among 162 bottles with Gram-positive organisms and 200 bottles with Gram-negative organisms, the MALDI Biotyper correctly identified 145 (89.5\%) and 187 (93.5\%), respectively, to the genus level and $111(68.5 \%)$ and $161(80.5 \%)$, respectively, to the species level. Among 32 bottles with organisms that stained positive for yeasts, the results of identification to the species level for 23 (71.9\%) obtained by the MALDI Biotyper were compatible with those obtained by conventional phenotypic methods. Among the bottles with score values $\geq 1.500$, the MALDI Biotyper correctly identified $99.2 \%(124 / 125)$ of the bottles with Gram-positive organisms, $97.6 \%(166 / 170)$ of the bottles with Gram-negative organisms and $100 \%(5 / 5)$ of the bottles with yeasts to the genus level and $77.6 \%$ (97/125) of the bottles with Gram-positive organisms, $87.1 \%(148 / 170)$ of the bottles with Gram-negative organisms and $100.0 \%(5 / 5)$ of the bottles with yeasts to the species level.

Figure 2 shows the TAT of blood cultures for several of the main microorganisms (species with no. of isolates $\geq 5$ ) in positive blood cultures with $\geq 90 \%$ concordant identification results between the MALDI Biotyper in flagged blood cultures and conventional phenotypic methods. The mean TAT for species-level identification in flagged blood cultures by the MALDI Biotyper was $22.8 \mathrm{~h}$ (range, 10.6-89.4h) for S. aureus, $17.7 \mathrm{~h}$ (range, 5.3-97.5 h) for E. coli, $20.1 \mathrm{~h}$ (range, 10.8$65.9 \mathrm{~h}$ ) for K. pneumoniae, and $18.5 \mathrm{~h}$ (range, 12.7-22.6h) for P. aeruginosa. 


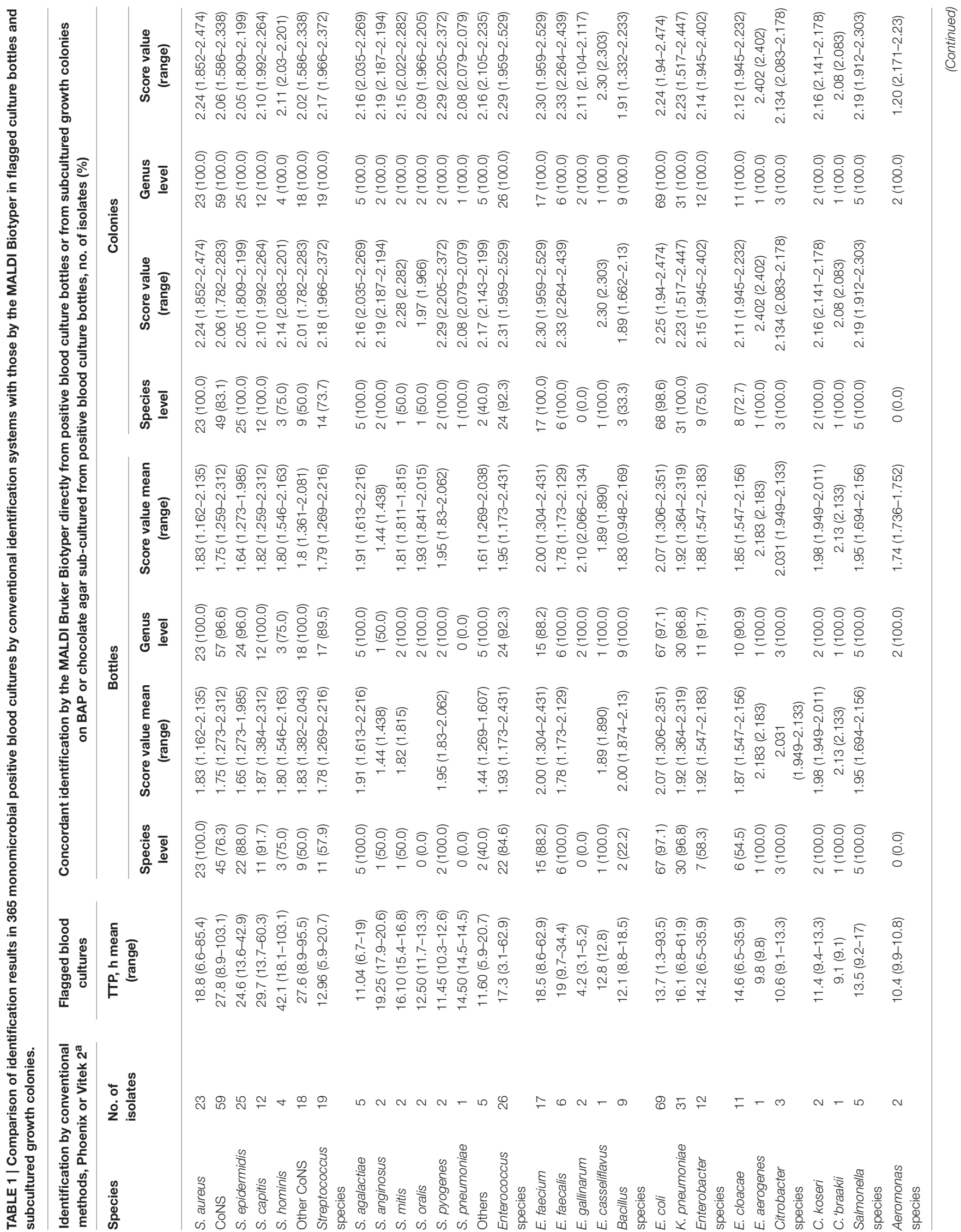




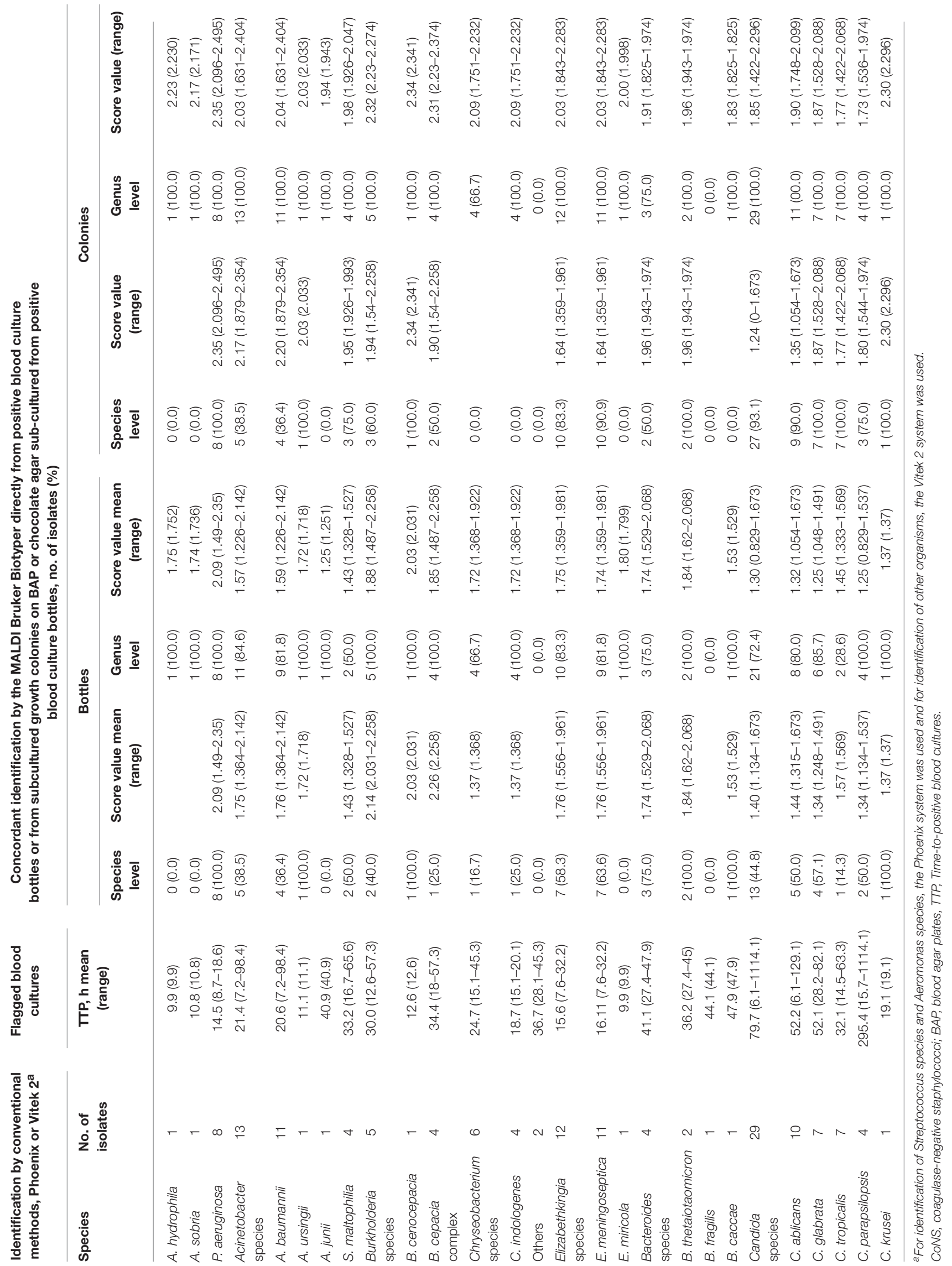


TABLE 2 | Summary of 37 incorrect identification results (types of identification VII, VIII, and IX) in monomicrobial flagged culture bottles by the MALDI Bruker Biotyper in comparison with those by the MALDI Biotyper in subcultured growth colonies from flagged culture bottles and conventional identification systems (see Figure 1 for description of identification types).

\begin{tabular}{|c|c|c|c|c|c|c|c|c|}
\hline \multirow[t]{2}{*}{ No. } & \multirow{2}{*}{$\begin{array}{l}\text { TTP } \\
\text { (h) }\end{array}$} & \multirow{2}{*}{$\begin{array}{l}\text { Gram staining } \\
\text { findings in positive } \\
\text { blood cultures }\end{array}$} & \multicolumn{4}{|c|}{ Identification results by MALDI Biotyper } & \multirow{2}{*}{$\begin{array}{l}\text { Identification results by } \\
\text { conventional methods }\end{array}$} & \multirow{2}{*}{$\begin{array}{l}\text { Patterns of } \\
\text { identification }\end{array}$} \\
\hline & & & $\begin{array}{l}\text { Bottle, organism (top } \\
\text { three matches) }\end{array}$ & $\begin{array}{l}\text { Score } \\
\text { value }\end{array}$ & $\begin{array}{l}\text { Colony, Organism } \\
\text { (best match) }\end{array}$ & $\begin{array}{l}\text { Score } \\
\text { value }\end{array}$ & & \\
\hline \multirow[t]{3}{*}{1.} & 11.5 & GNB & $\begin{array}{l}\text { Sphingobium } \\
\text { chlorophenolicum }\end{array}$ & 1.223 & $\begin{array}{l}\text { Acinetobacter } \\
\text { baumannii }\end{array}$ & 2.30 & A. baumannii & VII \\
\hline & & & $\begin{array}{l}\text { Aromatoleum } \\
\text { terpenicum }\end{array}$ & 1.133 & & & & \\
\hline & & & Moraxella bovis & 1.129 & & & & \\
\hline \multirow[t]{3}{*}{2.} & 22.1 & Yeasts & LactoB. sharpeae & 1.427 & Candida albicans & 1.79 & C. albicans & VII \\
\hline & & & L. sharpeae & 1.267 & & & & \\
\hline & & & L. crispatus & 1.247 & & & & \\
\hline \multirow[t]{3}{*}{3.} & 77.1 & Yeasts & A. terpenicum & 1.293 & C. albicans & 1.748 & C. albicans & VII \\
\hline & & & A. bremensis & 1.188 & & & & \\
\hline & & & Staphylococcus aureus & 1.128 & & & & \\
\hline \multirow[t]{3}{*}{4.} & 62.1 & Yeasts & A. anaerobicus & 1.159 & C. glabrata & 1.628 & C. glabrata & VII \\
\hline & & & A. anaerobicus & 1.140 & & & & \\
\hline & & & S. chlorophenolicum & 1.116 & & & & \\
\hline \multirow[t]{3}{*}{5.} & 15.7 & Yeasts & Riemerella columbina & 1.196 & C. tropicalis & 1.422 & C. tropicalis & VII \\
\hline & & & L. antri & 1.111 & & & & \\
\hline & & & C. tropicalis & 1.064 & & & & \\
\hline \multirow[t]{3}{*}{6.} & 22.1 & Yeasts & Penicillium digitatum & 1.135 & C. tropicalis & 1.756 & C. tropicalis & VII \\
\hline & & & C. albicans & 1.070 & & & & \\
\hline & & & C. albicans & 0.99 & & & & \\
\hline \multirow[t]{3}{*}{7.} & 63.3 & Yeasts & Pseudomonas putida & 1.235 & C. tropicalis & 1.591 & C. tropicalis & VII \\
\hline & & & A. ramosus & 1.126 & & & & \\
\hline & & & P. putida & 1.124 & & & & \\
\hline 8. & 58.2 & Yeasts & No peaks found & - & C. tropicalis & 1.671 & C. tropicalis & VII \\
\hline \multirow[t]{3}{*}{9.} & 14.5 & Yeasts & A. terpenicum & 1.347 & C. tropicalis & 2.068 & C. tropicalis & VII \\
\hline & & & A. terpenicum & 1.302 & & & & \\
\hline & & & Arthrobacter bergerei & 1.270 & & & & \\
\hline \multirow[t]{3}{*}{10.} & 61.6 & GPB & $\begin{array}{l}\text { Mannheimia } \\
\text { haemolytica }\end{array}$ & 1.172 & $\begin{array}{l}\text { Corynebacterium } \\
\text { riegelii }\end{array}$ & 2.099 & C. riegelii & VII \\
\hline & & & P. savastanoi & 1.158 & & & & \\
\hline & & & Bacillus wakoensis & 1.119 & & & & \\
\hline \multirow[t]{3}{*}{11.} & 18.3 & GNB & Weissella halotolerans & 1.367 & Elizabethkingia & 2.014 & E. meningoseptica & VII \\
\hline & & & $\begin{array}{l}\text { Flavobacterium } \\
\text { johnsoniae }\end{array}$ & 1.266 & meningoseptica & & & \\
\hline & & & L. gastricus & 1.248 & & & & \\
\hline \multirow[t]{3}{*}{12.} & 19.6 & GNB & P. savastanoi & 1.395 & E. miricola & 1.849 & E. meningoseptica & VII \\
\hline & & & $\begin{array}{l}\text { Stenotrophomonas } \\
\text { maltophilia }\end{array}$ & 1.162 & & & & \\
\hline & & & S. pasteuri & 1.157 & & & & \\
\hline 13. & 15.1 & GNB & M. haemolytica & 1.233 & $\begin{array}{l}\text { Enterobacter } \\
\text { cloacae }\end{array}$ & 1.945 & E. cloacae & VII \\
\hline
\end{tabular}




\section{TABLE 2 | Continued}

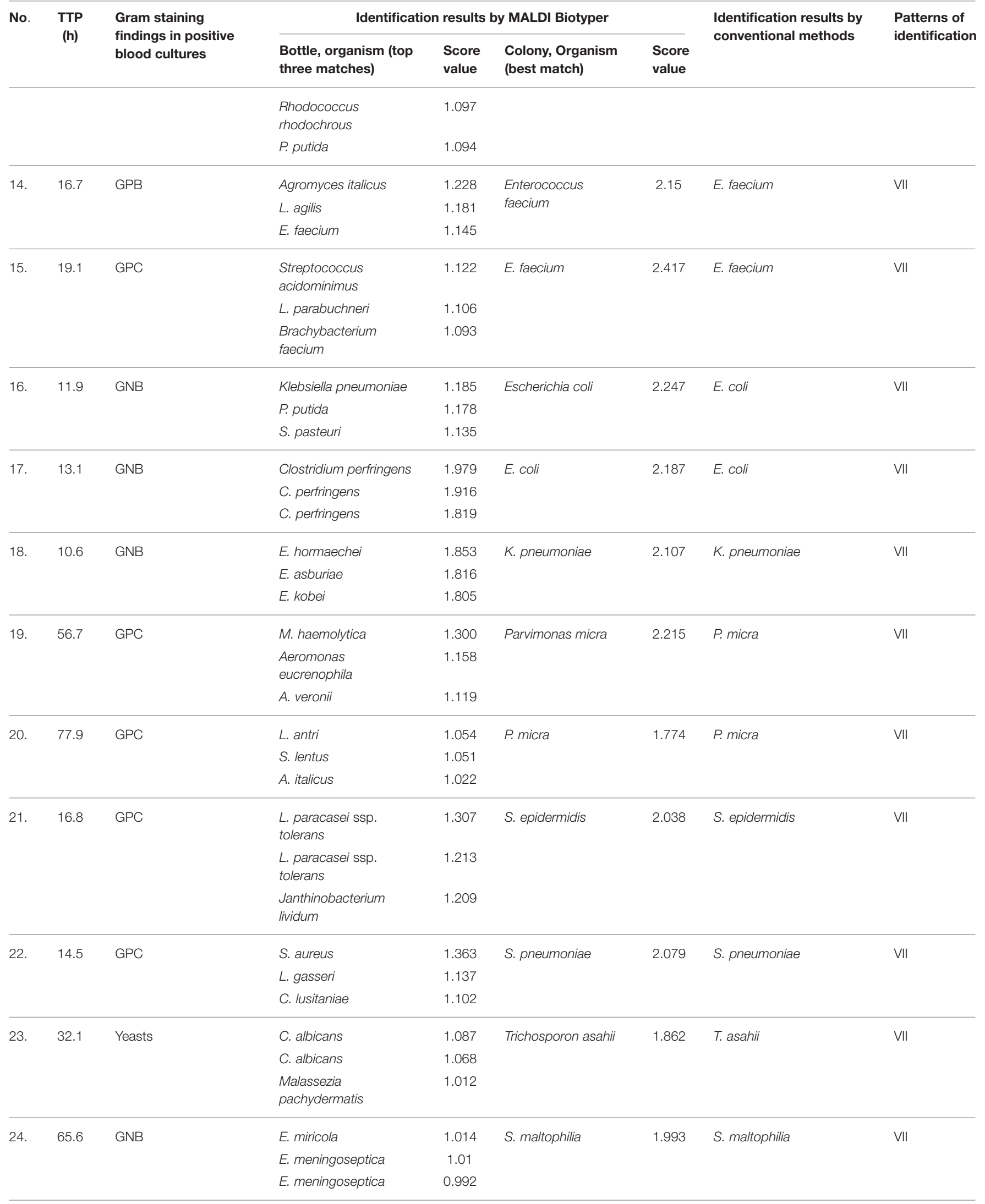




\section{TABLE 2 | Continued}

\begin{tabular}{|c|c|c|c|c|c|c|c|c|}
\hline \multirow[t]{2}{*}{ No. } & \multirow{2}{*}{$\begin{array}{l}\text { TTP } \\
\text { (h) }\end{array}$} & \multirow{2}{*}{$\begin{array}{l}\text { Gram staining } \\
\text { findings in positive } \\
\text { blood cultures }\end{array}$} & \multicolumn{4}{|c|}{ Identification results by MALDI Biotyper } & \multirow{2}{*}{$\begin{array}{l}\text { Identification results by } \\
\text { conventional methods }\end{array}$} & \multirow{2}{*}{$\begin{array}{l}\text { Patterns of } \\
\text { identification }\end{array}$} \\
\hline & & & $\begin{array}{l}\text { Bottle, organism (top } \\
\text { three matches) }\end{array}$ & $\begin{array}{l}\text { Score } \\
\text { value }\end{array}$ & $\begin{array}{l}\text { Colony, Organism } \\
\text { (best match) }\end{array}$ & $\begin{array}{l}\text { Score } \\
\text { value }\end{array}$ & & \\
\hline \multirow[t]{3}{*}{25.} & 18.5 & GPB & Mycobacterium szulgai & 1.123 & C. striatum & 2.343 & C. species & VIII \\
\hline & & & C. dubliniensis & 1.106 & & & & \\
\hline & & & A. bremensis & 1.102 & & & & \\
\hline \multirow[t]{3}{*}{26.} & 92.5 & GPC & S. aureus & 1.006 & M. luteus & 2.181 & M. species & VIII \\
\hline & & & S. aureus & 1.003 & & & & \\
\hline & & & S. pasteuri & 0.995 & & & & \\
\hline \multirow[t]{3}{*}{27.} & 82.7 & GPC & A. terpenicum & 1.444 & Micrococcus luteus & 2.217 & M. species & VIII \\
\hline & & & A. terpenicum & 1.242 & & & & \\
\hline & & & Azoarcus species & 1.232 & & & & \\
\hline \multirow[t]{3}{*}{28.} & 46.7 & Not visible & P. savastanoi & 1.265 & M. luteus & 2.005 & M. species & VIII \\
\hline & & & P. putida & 1.15 & & & & \\
\hline & & & P. congelana & 1.064 & & & & \\
\hline \multirow[t]{3}{*}{29.} & 15 & GNB & S. chlorophenolicum & 1.185 & A. baylyi & 1.742 & A. baumannii & VIII \\
\hline & & & C. chauvoei & 1.153 & & & & \\
\hline & & & A. radioresistens & 1.102 & & & & \\
\hline \multirow[t]{3}{*}{30.} & 103.1 & GPC & A. bremensis & 1.449 & S. pettenkoferi & 2.03 & S. hominis & VIII \\
\hline & & & A. terpenicum & 1.436 & & & & \\
\hline & & & L. sharpeae & 1.29 & & & & \\
\hline \multirow[t]{3}{*}{31.} & 31.2 & GNB & M. canis & 0.971 & S. rhizophila & 2.047 & S. maltophilia & VIII \\
\hline & & & M. canis & 0.947 & & & & \\
\hline & & & S. intermedius & 0.938 & & & & \\
\hline \multirow[t]{3}{*}{32.} & 44.1 & GPB & L. crispatus & 1.266 & Thauera aromatica & 1.285 & Bacteroides fragilis & IX \\
\hline & & & Tissierella praeacuta & 1.225 & & & & \\
\hline & & & P. brassicacearum & 1.208 & & & & \\
\hline \multirow[t]{3}{*}{33.} & 45.3 & GNB & P. putida & 1.185 & S. rhizophila & 2.062 & Chryseobacterium & IX \\
\hline & & & L. sharpeae & 1.114 & & & species & \\
\hline & & & L. alimentaurius & 1.102 & & & & \\
\hline \multirow[t]{3}{*}{34.} & 28.1 & GPB & T. aromatica & 1.369 & A. cumminsii & 1.849 & Chryseobacterium & IX \\
\hline & & & A. cumminsii & 1.276 & & & species & \\
\hline & & & S. faeni & 1.271 & & & & \\
\hline \multirow[t]{3}{*}{35.} & 33.2 & GPB & S. herbicidovorans & 1.167 & A. polychromogenes & 1.813 & C. species & IX \\
\hline & & & $\begin{array}{l}\text { Sphingomonas } \\
\text { adhaesiva }\end{array}$ & 1.126 & & & & \\
\hline & & & S. adhaesiva & 1.126 & & & & \\
\hline \multirow[t]{3}{*}{36.} & 78.5 & GPC & L. mucosae & 1.31 & Propionibacterium & 1.422 & Eggerthella lenta & IX \\
\hline & & & A. globiformis & 1.305 & avidum & & & \\
\hline & & & P. antarctica & 1.267 & & & & \\
\hline \multirow[t]{3}{*}{37.} & 79.8 & GPB & $\begin{array}{l}\text { Actinomyces } \\
\text { graevenitzii }\end{array}$ & 1.099 & A. nosocomialis & 1.594 & E. lenta & IX \\
\hline & & & R. erythropolis & 1.062 & & & & \\
\hline & & & Vibrio mimicus & 1.042 & & & & \\
\hline
\end{tabular}

TTP, time-to-positive blood culture; GPC, Gram-positive cocci; GPB, Gram-positive bacilli; GNB, Gram-negative bacilli. 


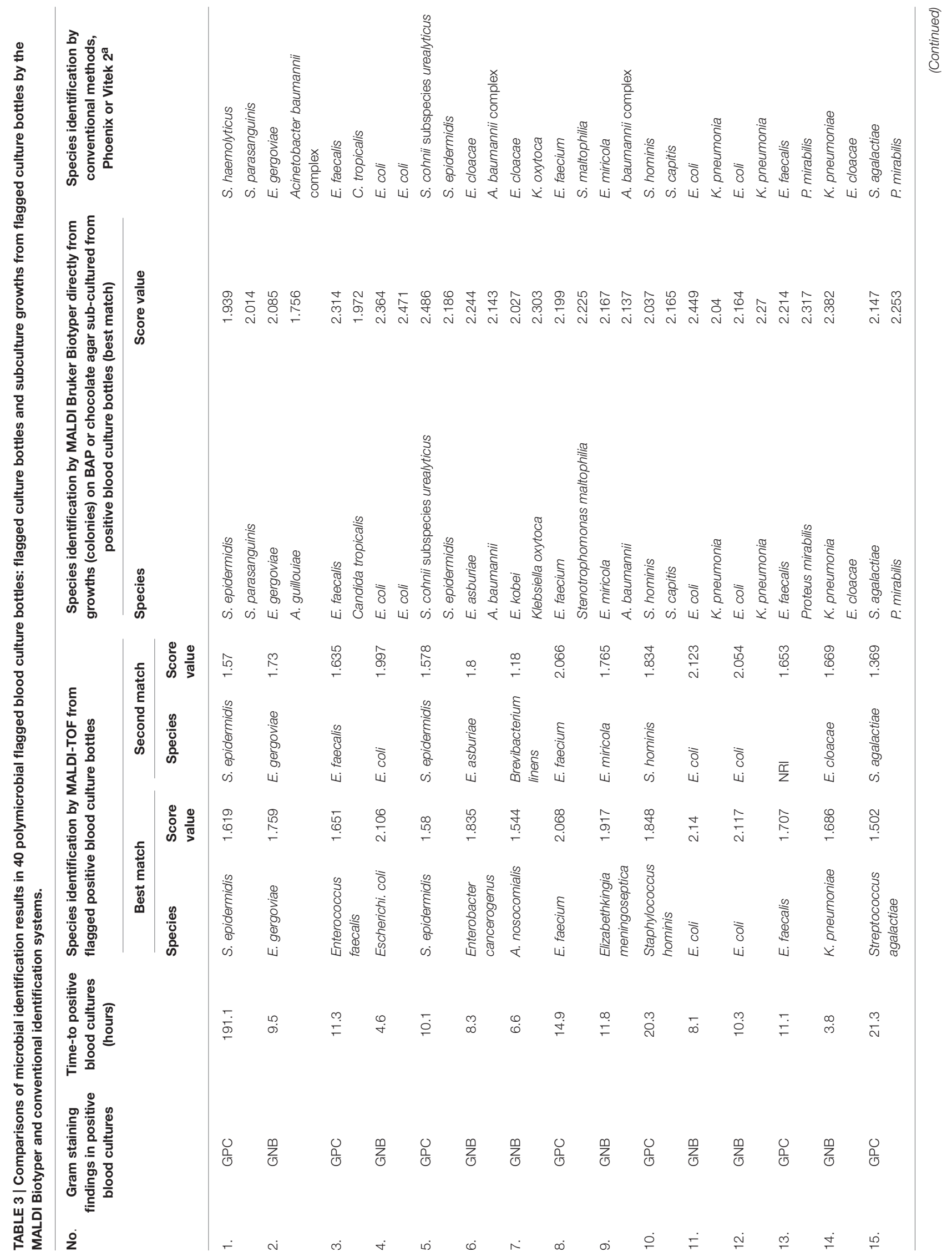



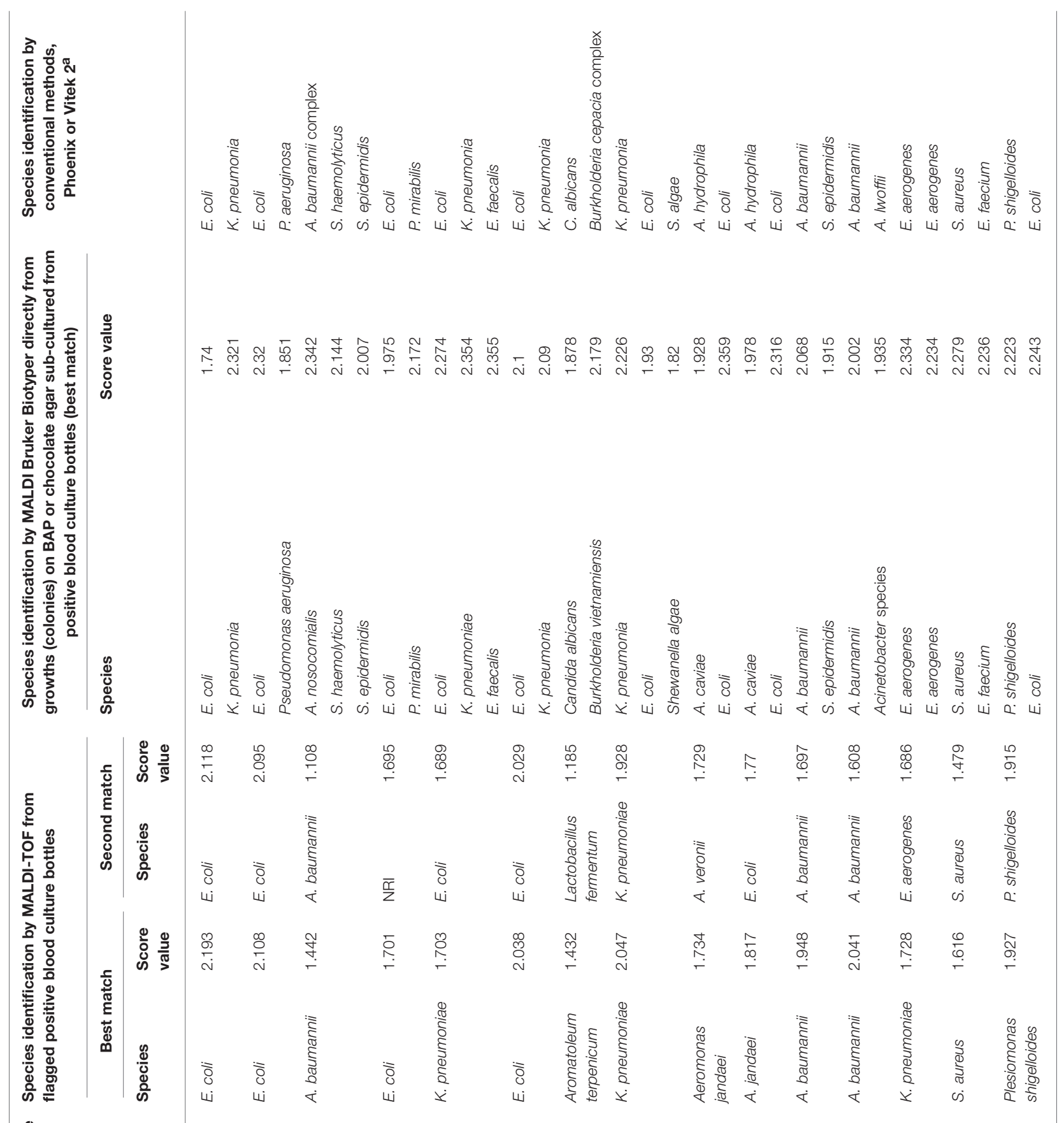

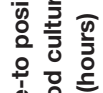

현

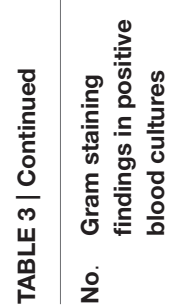

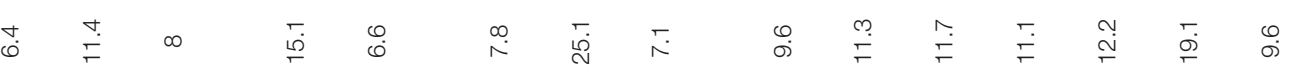

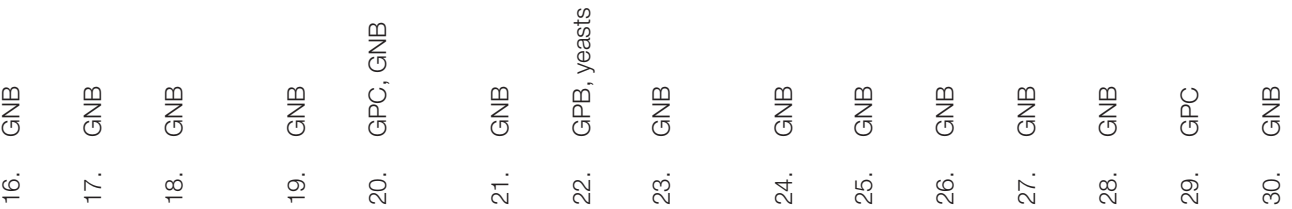




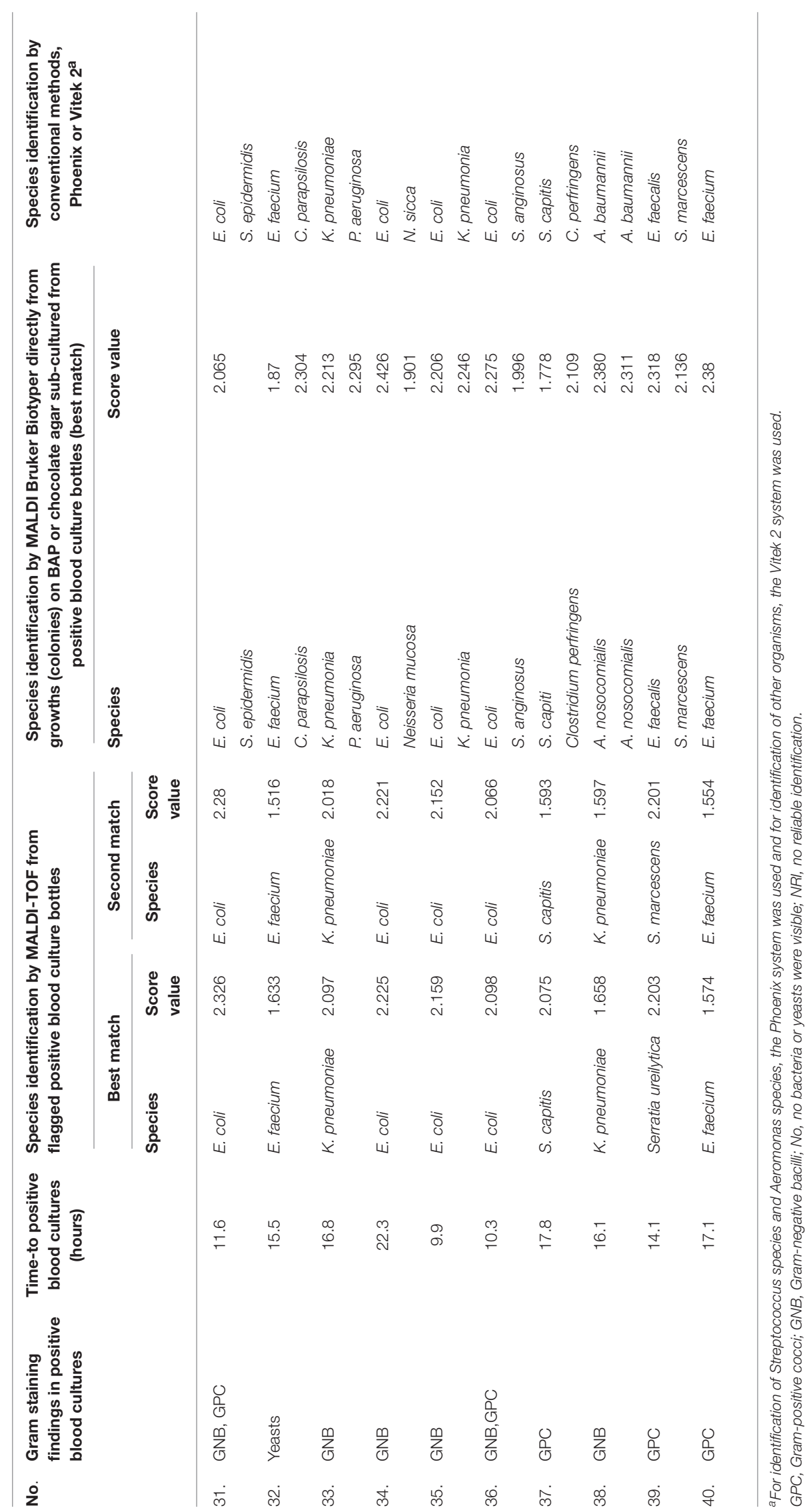




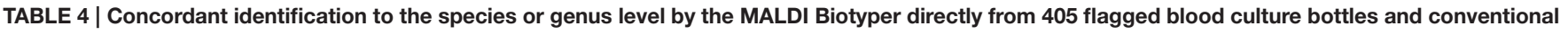
identification systems according to Gram staining results and identification scores.

\begin{tabular}{|c|c|c|c|c|c|c|c|c|c|c|c|c|}
\hline \multirow{3}{*}{$\begin{array}{l}\text { Identification } \\
\text { scores value }\end{array}$} & \multicolumn{12}{|c|}{ Gram stain findings in positive blood cultures } \\
\hline & \multicolumn{3}{|c|}{ Gram-positive } & \multicolumn{3}{|c|}{ Gram-negative } & \multicolumn{3}{|c|}{ Yeast } & \multicolumn{3}{|c|}{ Other ${ }^{a}$} \\
\hline & No. & $\begin{array}{c}\text { Species } \\
\text { level no. } \\
(\%)\end{array}$ & $\begin{array}{c}\text { Genus } \\
\text { level no. } \\
(\%)\end{array}$ & No. & $\begin{array}{c}\text { Species } \\
\text { level no. } \\
(\%)\end{array}$ & $\begin{array}{c}\text { Genus level } \\
\text { no. (\%) }\end{array}$ & No. & $\begin{array}{c}\text { Species } \\
\text { level no. } \\
\text { (\%) }\end{array}$ & $\begin{array}{c}\text { Genus } \\
\text { level no. } \\
(\%)\end{array}$ & No. & $\begin{array}{c}\text { Species } \\
\text { level no. } \\
(\%)\end{array}$ & $\begin{array}{c}\text { Genus } \\
\text { level no. } \\
(\%)\end{array}$ \\
\hline All & 162 & $111(68.5)$ & $145(89.5)$ & 200 & $161(80.5)$ & $187(93.5)$ & 32 & $16(50.0)$ & $23(71.9)$ & 11 & $8(72.7)$ & $9(81.8)$ \\
\hline$<1.399$ & 30 & $11(36.7)$ & $16(53.3)$ & 21 & $6(28.6)$ & $12(57.1)$ & 24 & $14(1.7)$ & $16(66.7)$ & 3 & 1 (33.3) & $2(66.7)$ \\
\hline $1.400-1.499$ & 7 & $3(42.9)$ & $5(71.4)$ & 9 & $7(77.8)$ & $9(100.0)$ & 3 & 1 (33.3) & $2(66.7)$ & 1 & $0(0.0)$ & $0(0.0)$ \\
\hline $1.500-1.599$ & 15 & $12(80.0)$ & 14 (93.3) & 10 & 7 (70.0) & $9(90.0)$ & 3 & 3 (100.0) & 3 (100.0) & 2 & $2(100.0)$ & 2 (100.0) \\
\hline $1.600-1.699$ & 19 & $14(73.7)$ & 19 (100.0) & 7 & $6(85.7)$ & $6(85.7)$ & 2 & $2(100.0)$ & $2(100.0)$ & 0 & - & - \\
\hline $1.700-1.999$ & 48 & 37 (77.1) & 48 (100.0) & 57 & 41 (71.9) & 55 (96.5) & 0 & - & - & 2 & $2(100.0)$ & $2(100.0)$ \\
\hline$\geqq 2.000$ & 43 & 34 (79.1) & 43 (100.0) & 96 & $94(97.9)$ & 96 (100.0) & 0 & - & - & 3 & 3 (100.0) & $3(100.0)$ \\
\hline$\geqq 1.400$ & 132 & $100(75.8)$ & $129(97.7)$ & 179 & 155 (86.6) & $175(97.8)$ & 8 & $6(75.0)$ & 7 (87.5) & 8 & 7 (87.5) & $7(87.5)$ \\
\hline$\geqq 1.500$ & 125 & $97(77.6)$ & $124(99.2)$ & 170 & $148(87.1)$ & $166(97.6)$ & 5 & 5 (100.0) & $5(100.0)$ & 7 & $7(100.0)$ & 7 (100.0) \\
\hline$\geqq 1.600$ & 110 & 85 (77.3) & 110 (100.0) & 160 & $141(88.1)$ & $157(98.1)$ & 2 & $2(100.0)$ & 2 (100.0) & 5 & 5 (100.0) & 5 (100.0) \\
\hline$\geqq 1.700$ & 91 & 71 (78.0) & 91 (100.0) & 153 & 135 (88.2) & 151 (98.7) & 0 & - & - & 5 & 5 (100.0) & 5 (100.0) \\
\hline
\end{tabular}

${ }^{a}$ No bacteria or yeasts were visible or no mixed organisms were found.

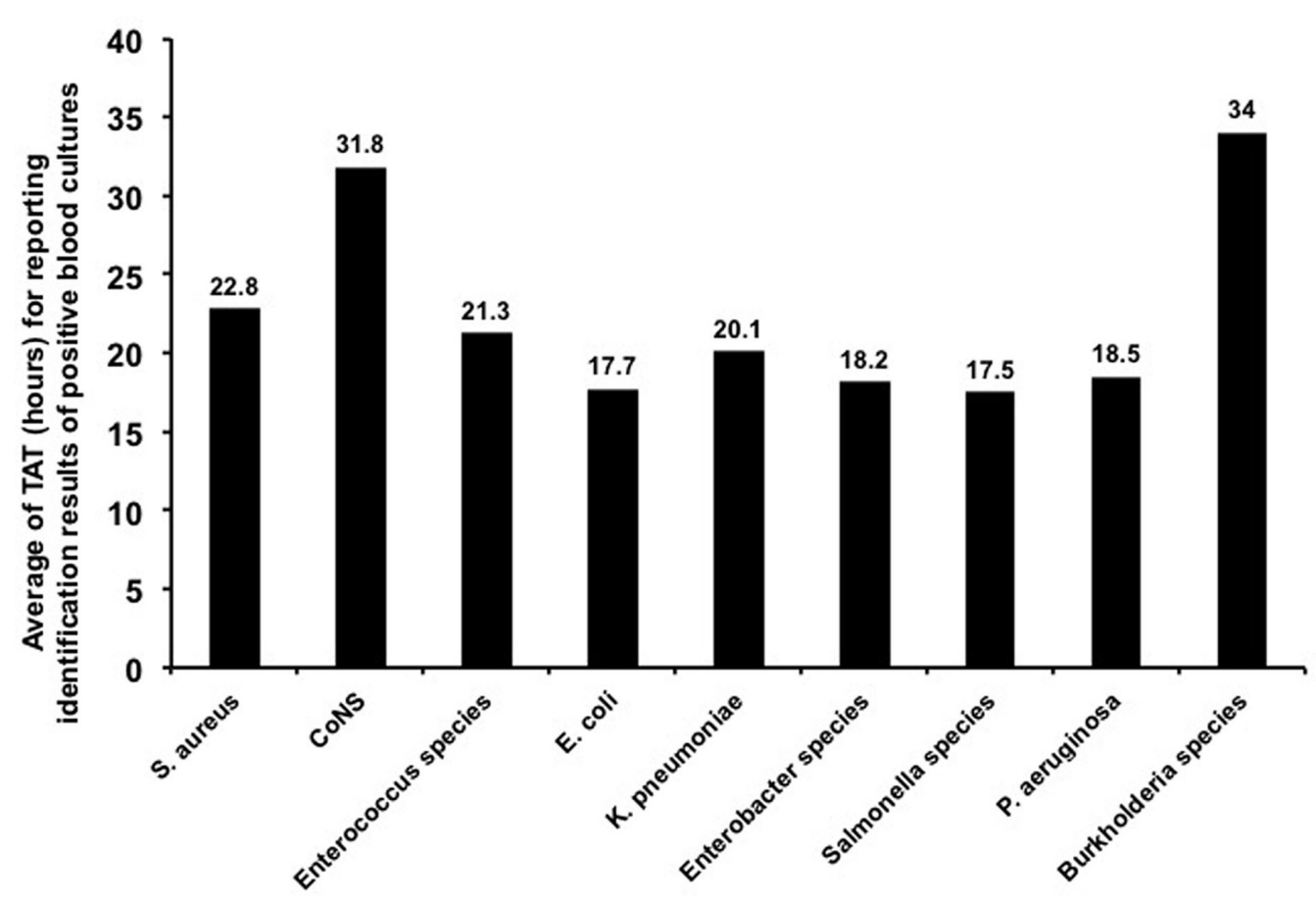

FIGURE 2 | Turn-around time (TAT) of positive blood cultures. TAT of blood cultures for several main microorganisms (no. of isolates $\geq 5$ ) from positive blood cultures with concordant identification results of $\geq 90 \%$ with conventional phenotypic methods. TAT of blood cultures was defined as the time interval between blood cultures collected from patients and laboratory reporting of identification results from positive blood cultures.

\section{DISCUSSION}

We used an in-house saponin-based extraction method to evaluate the performance of the Bruker Biotyper MALDI-TOF/MS system for the identification of bacteria and fungi in 405 positively flagged blood culture bottles. Results obtained from MALDI-TOF/MS were compared with those obtained using conventional phenotypic identification methods. Among 365 bottles with monomicrobal growth, the microorganisms were correctly identified to the species $(72.1 \%)$ 
or genus (89.6\%) level. Moreover, among 40 polymicrobial cultures, the MALDI Biotyper correctly identified at least one organism to the species level in 33 (82.5\%) positive blood culture bottles and to the genus level in 37 (92.5\%) bottles.

MALDI-TOF MS is a useful modality for identifying bacterial species directly from positive blood cultures. For accurate results, however, protein extraction methods are necessary to reduce the number of background peaks caused by nonbacterial proteins in blood culture bottles. Schubert et al. (2011) and Juiz et al. (2012) reported that the Sepsityper kit was superior to centrifugation methods for protein extraction in BACTEC bottles. More recently, some studies have demonstrated that saponin-based extraction leads to results identical to if not better than those obtained by the Sepsityper kit. Using BacT/ALERT anaerobic positive blood cultures, Meex et al. (2012) reported no significant difference in the rates of correct species-level identification between the Sepsityper kit (67\%) and a saponin-based extraction method (66\%). Working from BACTEC bottles, Martiny et al. also found that the rates of correct species-level identification from positive blood culture bottles were similar between the Sepsityper kit (68.4\%) and an inhouse saponin-based extraction method (73.7\%) (Martiny et al., 2012).

Rapid identification of organisms to the genus level is essential, especially for organisms with predictable resistance (such as Enterobacter sp., Acinetobacter sp., Pseudomonas sp., and Stenotrophomonas sp.) (Chen et al., 2013; Davey et al., 2013; Huang et al., 2013; Nagel et al., 2014). We found that the MALDI Biotyper provided good genus-level identification results. The sensitivity of the system for correctly identifying pathogens to the genus level was $89.6 \%$ in monomicrobal cultures, and the overall accuracy of identification to the genus level in positive blood cultures was $89.5 \%$ for Grampositive organisms, $93.5 \%$ for Gram-negative organisms and $71.9 \%$ for yeasts. These findings are in agreement with those reported in previous studies, which showed that the accuracy of MALDI-TOF was higher for Gram-negative organisms than for Gram-positive organisms (Lagace-Wiens et al., 2012; Meex et al., 2012).

The rate of correct identification by MALDI-TOF MS is largely dependent on confidence score. Among the 405 positive blood culture bottles investigated in this study, 307 (75.8\%) had confidence scores $\geqq 1.500,249$ (61.5\%) had scores $\geqq 1.700$ and $142(35.1 \%)$ had confidence scores $\geqq 2.000$; none of the yeast cultures had scores $\geqq 1.700$. With a cutoff score of $\geqq 1.700$ (Bruker's recommended criteria for genus identification), the MALDI Biotyper correctly identified $78.0 \%$ of Gram-positive organisms and $88.2 \%$ of Gram-negative organisms to the species level and 100 and $98.7 \%$, respectively, to the genus level. In a recent study, Lagace-Wiens et al. found that the cutoff values could be lowered without compromising accuracy when MALDITOF is applied directly to positive blood cultures (Lagace-Wiens et al., 2012). Working from BACTEC bottles, Schubert et al. (2011) also demonstrated the possibility of accepting specieslevel identifications in blood culture bottles with low scores $(\geqq 1.500)$ if the first three proposed results were identical. In
BacT/ALERT anaerobic blood culture bottles, Meex et al. further showed that Bruker's recommended criteria could be expanded to avoid the exclusion of a significant percentage of correct identifications, mainly among Gram-positive bacteria (Meex et al., 2012). Similarly, with a lower cutoff score of 1.500 , we found that the MALDI Biotyper could correctly identify Gram-positive organisms, Gram-negative organisms and yeasts to the species (77.6, 87.1, and $100 \%$, respectively) or genus level (99.2, 97.6, and $100 \%$, respectively) in blood culture bottles. Thus, for identification to the genus level, we propose that the cutoff value can be lowered to $\geqq 1.500$ without compromising the reliability of the identification results (accuracy $>95 \%$ ).

Although some studies have reported that MALDI-TOF can accurately identify yeasts to the species level (Spanu et al., 2012; Won et al., 2013), we found that most blood cultures containing yeast isolates had low confidence scores and lower rates of correct identification than blood cultures containing bacteria only. Buchan et al reported similar findings (Buchan et al., 2012). This may be because the extraction protocol used in our study and in that by Buchan et al was not optimized for recovery of yeasts. However, with a modified cut off value of 1.500 , yeasts in all 5 bottles with confidence scores $\geqq 1.500$ were correctly identified to the species level. An additional extraction step would be required to obtain more reliable identification results for yeasts with confidence scores $<1.500$ (Martiny et al., 2012).

In polymicrobial cultures, MALDI-TOF MS typically yields a single identification confidence score for the pre-dominant species (Lagace-Wiens et al., 2012) and is unable to identify multiple organisms. Although one study reported that it may be possible to identify multiple organisms with different Gram staining reactions in polymicrobial cultures (Ferroni et al., 2010), we found that the MALDI Biotyper correctly identified only two organisms in $2(5.0 \%)$ bottles among 40 polymicrobial cultures. In contrast, organisms in a polymicrobial culture could be identified to the species level in 34 bottles (85.0\%) from subcultures on BAP. This emphasizes the importance of subculturing positive cultures for definitive identification of organisms in polymicrobial cultures.

Rapid identification of bloodstream pathogens is important so that appropriate antibiotic treatment can be administered (Vlek et al., 2012). Using the MALDI Biotyper to identify specimens prepared by the Sepsityper kit, Buchan et al. found that the median TAT (from blood collection to species identification) was 23-83 $\mathrm{h}$ faster than routine methods for Gram-positive isolates and $34-51 \mathrm{~h}$ faster for Gram-negative isolates (Buchan et al., 2012). Egli et al. also found that the median TAT was 27.4 (25.8-29.3) h using MALDI-TOF (Egli et al., 2015). With our inhouse saponin-based extraction protocol, we found that the mean TAT for identification of Gram-positive organisms (S. aureus, coagulase-negative staphylococci and Enterococcus species) was 21-32 $\mathrm{h}$ and that the mean TAT for identification of Gramnegative isolates was less than $20 \mathrm{~h}$. The TAT of conventional phenotypic identification methods from sub-cultured colonies were 37-56 h for Gram-positive isolates and 36-52 h for Gramnegative isolates which were 16-24h longer than that of MALDI Biotyper system. 


\section{SUMMARY}

In this study, we evaluated the performance of the MALDI Biotyper with an in-house saponin-based method for routine identification of isolates directly from positive blood culture bottles. Our protocol yielded a genus-level identification rate of $89.9 \%(364 / 405)$ and a species-level identification rate of $73.1 \%$ (296/405). Confidence scores for yeasts were significantly lower than those for Gram-positive and Gramnegative isolates and compromised the accuracy of identification. Further optimization of the protein extraction procedure

\section{REFERENCES}

Bassetti, M., Merelli, M., Ansaldi, F., de Florentiis, D., Sartor, A., Scarparo, C., et al. (2015). Clinical and therapeutic aspects of candidemia: a five year single centre study. PLOS ONE 10:e127534. doi: 10.1371/journal.pone. 0127534

Buchan, B. W., Riebe, K. M., and Ledeboer, N. A. (2012). Comparison of the MALDI Biotyper system using Sepsityper specimen processing to routine microbiological methods for identification of bacteria from positive blood culture bottles. J. Clin. Microbiol. 50, 346-352. doi: 10.1128/JCM. 05021-11

Chen, J. H., Ho, P. L., Kwan, G. S., She, K. K., Siu, G. K., Cheng, V. C., et al. (2013). Direct bacterial identification in positive blood cultures by use of two commercial matrix-assisted laser desorption ionization-time of flight mass spectrometry systems. J. Clin. Microbiol. 51, 1733-1739. doi: 10.1128/JCM.03259-12

Davey, P., Brown, E., Charani, E., Fenelon, L., Gould, I. M., Holmes, A., et al. (2013). Interventions to improve antibiotic prescribing practices for hospital inpatients. Cochrane Database Syst. Rev. 4:CD003543. doi: 10.1002/14651858.CD003543.pub3

Deen, J., von Seidlein, L., Andersen, F., Elle, N., White, N. J., and Lubell, Y. (2012). Community-acquired bacterial bloodstream infections in developing countries in south and southeast Asia: a systematic review. Lancet Infect. Dis. 12, 480-487. doi: 10.1016/S1473-3099(12)70028-2

Egli, A., Osthoff, M., Goldenberger, D., Halter, J., Schaub, S., Steiger, J., et al. (2015). Matrix-assisted laser desorption/ionization time-offlight mass spectrometry (MALDI-TOF) directly from positive blood culture flasks allows rapid identification of bloodstream infections in immunosuppressed hosts. Transpl. Infect. Dis. 17, 481-487. doi: 10.1111/tid. 12373

Ferroni, A., Suarez, S., Beretti, J. L., Dauphin, B., Bille, E., Meyer, J., et al. (2010). Real-time identification of bacteria and Candida species in positive blood culture broths by matrix-assisted laser desorption ionization-time of flight mass spectrometry. J. Clin. Microbiol. 48, 1542-1548. doi: 10.1128/JCM. 02485-09

Huang, A. M., Newton, D., Kunapuli, A., Gandhi, T. N., Washer, L. L., Isip, J., et al. (2013). Impact of rapid organism identification via matrix-assisted laser desorption/ionization time-of-flight combined with antimicrobial stewardship team intervention in adult patients with bacteremia and candidemia. Clin. Infect. Dis. 57, 1237-1245. doi: 10.1093/cid/ cit498

Juiz, P. M., Almela, M., Melcion, C., Campo, I., Esteban, C., Pitart, C., et al. (2012). A comparative study of two different methods of sample preparation for positive blood cultures for the rapid identification of bacteria using MALDI-TOF MS. Eur. J. Clin. Microbiol. Infect. Dis. 31, 1353-1358. doi: 10.1007/s10096-011-1449-x

Lagace-Wiens, P. R., Adam, H. J., Karlowsky, J. A., Nichol, K. A., Pang, P. F., Guenther, J., et al. (2012). Identification of blood culture isolates directly from positive blood cultures by use of matrix-assisted laser desorption ionizationtime of flight mass spectrometry and a commercial extraction system: analysis of performance, cost, and turnaround time. J. Clin. Microbiol. 50, 3324-3328. doi: 10.1128/JCM.01479-12 in positive blood cultures is needed to improve the rate of identification.

\section{AUTHOR CONTRIBUTIONS}

JC, TL, and SD conceived and designed the experiments, performed the experiments, analyzed the data, and wrote the paper. JC, TL, SD, ST, CL, and WS performed the experiments and analyzed the data. JC, TL, SD, ST, CL, WS, and $\mathrm{PH}$ read and approved the final version of the manuscript.

Lai, C. C., Chen, Y. H., Lin, S. H., Chung, K. P., Sheng, W. H., Ko, W. C., et al. (2014). Changing aetiology of healthcare-associated bloodstream infections at three medical centres in Taiwan, 2000-2011. Epidemiol. Infect. 142, 2180-2185. doi: $10.1017 /$ S0950268813003166

Loonen, A. J., Jansz, A. R., Stalpers, J., Wolffs, P. F., and van den Brule, A. J. (2012). An evaluation of three processing methods and the effect of reduced culture times for faster direct identification of pathogens from BacT/ALERT blood cultures by MALDI-TOF MS. Eur. J. Clin. Microbiol. Infect. Dis. 31, 1575-1583. doi: 10.1007/s10096-011-1480-y

March-Rossello, G. A., Munoz-Moreno, M. F., Garcia-Loygorri-Jordan de Urries, M. C., and Bratos-Perez, M. A. (2013). A differential centrifugation protocol and validation criterion for enhancing mass spectrometry (MALDITOF) results in microbial identification using blood culture growth bottles. Eur. J. Clin. Microbiol. Infect. Dis. 32, 699-704. doi: 10.1007/s10096-01 2-1797-1

Martiny, D., Debaugnies, F., Gateff, D., Gerard, M., Aoun, M., Martin, C., et al. (2013). Impact of rapid microbial identification directly from positive blood cultures using matrix-assisted laser desorption/ionization time-of-flight mass spectrometry on patient management. Clin. Microbiol. Infect. 19, E568-E581. doi: 10.1111/1469-0691.12282

Martiny, D., Dediste, A., and Vandenberg, O. (2012). Comparison of an in-house method and the commercial Sepsityper kit for bacterial identification directly from positive blood culture broths by matrixassisted laser desorption-ionisation time-of-flight mass spectrometry. Eur. J. Clin. Microbiol. Infect. Dis. 31, 2269-2281. doi: 10.1007/s10096-01 2-1566-1

Meex, C., Neuville, F., Descy, J., Huynen, P., Hayette, M. P., De Mol, P., et al. (2012). Direct identification of bacteria from BacT/ALERT anaerobic positive blood cultures by MALDI-TOF MS: MALDI Sepsityper kit versus an in-house saponin method for bacterial extraction. J. Med. Microbiol. 61, 1511-1516. doi: 10.1099/jmm.0.044750-0

Moussaoui, W., Jaulhac, B., Hoffmann, A. M., Ludes, B., Kostrzewa, M., Riegel, P., et al. (2010). Matrix-assisted laser desorption ionization time-of-flight mass spectrometry identifies $90 \%$ of bacteria directly from blood culture vials. Clin. Microbiol. Infect. 16, 1631-1638. doi: 10.1111/j.1469-0691.2010.03356.x

Nagel, J. L., Huang, A. M., Kunapuli, A., Gandhi, T. N., Washer, L. L., Lassiter, J., et al. (2014). Impact of antimicrobial stewardship intervention on coagulase-negative Staphylococcus blood cultures in conjunction with rapid diagnostic testing. J. Clin. Microbiol. 52, 2849-2854. doi: 10.1128/JCM. 00682-14

Saffert, R. T., Cunningham, S. A., Mandrekar, J., and Patel, R. (2012). Comparison of three preparatory methods for detection of bacteremia by MALDITOF mass spectrometry. Diagn. Microbiol. Infect. Dis. 73, 21-26. doi: 10.1016/j.diagmicrobio.2012.01.010

Schubert, S., Weinert, K., Wagner, C., Gunzl, B., Wieser, A., Maier, T., et al. (2011). Novel, improved sample preparation for rapid, direct identification from positive blood cultures using matrix-assisted laser desorption/ionization time-of-flight (MALDI-TOF) mass spectrometry. J. Mol. Diagn. 13, 701-706. doi: 10.1016/j.jmoldx.2011.07.004

Spanu, T., Posteraro, B., Fiori, B., D’Inzeo, T., Campoli, S., Ruggeri, A., et al. (2012). Direct maldi-tof mass spectrometry assay of blood culture broths for rapid identification of Candida species causing bloodstream infections: an 
observational study in two large microbiology laboratories. J. Clin. Microbiol. 50, 176-179. doi: 10.1128/JCM.05742-11

Vlek, A. L., Bonten, M. J., and Boel, C. H. (2012). Direct matrix-assisted laser desorption ionization time-of-flight mass spectrometry improves appropriateness of antibiotic treatment of bacteremia. PLoS ONE 7:e32589. doi: 10.1371/journal.pone.0032589

Won, E. J., Shin, J. H., Lee, K., Kim, M. N., Lee, H. S., Park, Y. J., et al. (2013). Accuracy of species-level identification of yeast isolates from blood cultures from 10 university hospitals in South Korea by use of the matrix-assisted laser desorption ionization-time of flight mass spectrometry-based Vitek MS system. J. Clin. Microbiol. 51, 3063-3065. doi: 10.1128/JCM.00945-13
Conflict of Interest Statement: The authors declare that the research was conducted in the absence of any commercial or financial relationships that could be construed as a potential conflict of interest.

Copyright (C) 2016 Chien, Lee, Du, Teng, Liao, Sheng, Teng and Hsueh. This is an open-access article distributed under the terms of the Creative Commons Attribution License (CC BY). The use, distribution or reproduction in other forums is permitted, provided the original author(s) or licensor are credited and that the original publication in this journal is cited, in accordance with accepted academic practice. No use, distribution or reproduction is permitted which does not comply with these terms. 